\title{
Demand Controlled Ventilation for Electric Demand Side Flexibility
}

\author{
Anthony Vautrin ${ }^{1,2}$, Sebastian Troitzsch ${ }^{1}$, \\ Srikkanth Ramachandran ${ }^{1}$, Thomas Hamacher ${ }^{3}$ \\ ${ }^{1}$ TUMCREATE Limited, Singapore \\ ${ }^{2}$ MINES ParisTech, Paris, France \\ ${ }^{3}$ Technical University of Munich (TUM), Garching, Germany
}

\begin{abstract}
The intermittency of renewable electricity generation and the increasing peak demand due to electric mobility pose challenges to the electric grid operation. Demand side flexibility (DSF) is becoming an important technique to counter these challenges, where the air-conditioning demand of commercial buildings has a particularly high potential to serve as a flexible load. This paper proposes demand controlled ventilation (DCV) as a method to increase the DSF of air-conditioned commercial buildings. Two DCV strategies are proposed based on (1) occupancy-based ventilation according to ASHRAE Standard 62.1 and (2) an indoor air quality (IAQ) model. The increase/changes in DSF are quantified for both the strategies. For a test case comprising a zone of an office building in Singapore, both DCV strategies are shown to increase the DSF when compared to a conservative ventilation strategy. For instance, the IAQbased DCV strategy allows for scheduling strategic reserves six-fold higher than the conservative ventilation strategy.
\end{abstract}

\section{Introduction}

The increasing share of non-controllable renewable energy sources in the electricity generation mix makes matching demand and generation capacities increasingly difficult. At the same time, the introduction of electric mobility could create peak loads that exceed the local grid hosting capacity. Demand side flexibility (DSF) is becoming an important tool to mitigate these challenges. In micro grids, DSF could lower the stress on storage facilities, thus decreasing their cost and improving feasibility (Atia and Yamada (2016)).

Studies have shown that commercial buildings can provide DSF through their heating, ventilation and air-conditioning (HVAC) systems, due to the inherent thermal energy storage capacities of buildings (Hanif et al. (2017)). The scheduling problem of the HVAC system can be formulated as an inter-temporal optimization problem aimed at minimizing the operational costs, i.e., energy costs, subject to comfort requirements, enabling DSF with indirect control through the energy price. However, the requirement to provide a fixed amount of outdoor air to ensure sufficient indoor air quality (IAQ), e.g., according to ASHRAE Standard 62.1, is observed to be a key limitation for load flexibility (fig. 3).

To this end, demand controlled ventilation (DCV) is proposed to increase DSF. As cooling and ventilation needs can make up to $60 \%$ of the energy consumption in commercial buildings in Singapore (Chua et al. (2013)), DCV was first developed as a way to reduce energy consumption by adapting the outdoor airflow rate to the current room conditions, while maintaining IAQ in a comfortable range (Laverge et al. (2011); Pantazaras et al. (2018); Ng et al. (2011)). However, as the ventilation requirements are alleviated, some DCV strategies enable ventilation loads to be shifted over time and may thus increase the DSF.

The main contribution of this paper is to analyze DCV as a method to increase the DSF of airconditioned commercial buildings. To this end, firstly a thermal building model is formulated along with an optimization framework that aims at minimizing the operation costs subject to comfort constraints. Secondly, two DCV strategies are proposed, based on (1) occupancy-based ventilation and (2) IAQ-based ventilation, with an IAQ model being derived from a grey clustering model of indoor air. Thirdly, numerical indicators for quantifying DSF are introduced, based on forced operation, price sensitivity and reserves scheduling. Lastly, a test case based on an office in Singapore is introduced. The change in DSF due to the DCV strategies is evaluated and discussed.

\section{Control framework}

This paper focuses on DSF in the HVAC systems of office buildings assuming a dynamic electricity tariff. This section first introduces the market model along with the optimal scheduling problem. Then, the mathematical formulation of the building model is developed.

\section{Optimal scheduling problem}

The National Electricity Market of Singapore (NEMS) is adopted in this work. As part of the Singapore Open Electricity Market initiative, all consumers will be able to purchase electricity at the half-hourly wholesale market rates. In the following is assumed 
that the energy price forecast $c_{t}^{\text {elc }}$ is perfectly known and does not depend on the building load schedule $\boldsymbol{u}_{t}$. The total cost of the building operation $j(\boldsymbol{u})$ is calculated on the basis of a discretization time step $\Delta t$, over a finite horizon $n_{t}$ :

$$
j(\boldsymbol{u})=\sum_{t \in \mathcal{T}} c_{t}^{e l c}\left\|\boldsymbol{u}_{t}\right\| \Delta t
$$

The optimal load schedule is obtained by minimizing the operation costs $j(\boldsymbol{u})$ over the horizon $\mathcal{T}$, with respect to the comfort and operational constraints $\boldsymbol{y}_{\min }$ and $\boldsymbol{y}_{\max }$. Therefore the numerical optimization problem can be expressed as:

$$
\begin{array}{ll}
\min _{\boldsymbol{u}_{t}} & j(\boldsymbol{u}) \\
\text { s.t. } & \boldsymbol{y}_{\text {min }} \leq \boldsymbol{y}_{t} \leq \boldsymbol{y}_{\text {max }}
\end{array}
$$

\section{Thermal building model}

In this work, the thermal comfort is expressed in terms of the indoor air temperature. Hence, the thermal building model expresses the relationship between the indoor air temperature, the electric load of the HVAC system, the local weather conditions and the building occupancy. The indoor air temperature, i.e. zone temperature, within each zone is assumed to be uniformly distributed.

The differential equation of the zone temperature $T_{z}$ of zone $z$ is expressed as:

$$
\frac{d T_{z}}{d t}=\frac{\dot{Q}_{z}}{C_{z}^{t h}} \quad \forall z \in \mathcal{Z}
$$

Where $\dot{Q}_{z}$ is the sum of all heat fluxes towards zone $z$ and $C_{z}^{t h}$ is the thermal heat capacity of zone $z$. The symbol $\mathcal{Z}$ is the set of all zones $z$. An equivalent formulation for the temperature $T_{s}$ of the surfaces $s$ is omitted here for the sake of brevity.

Ground heat transfer is omitted in the following, because the considered test case comprises the intermediate storey of an high-rise building, where adiabatic surfaces are assumed for ceiling and floor.

\section{Exterior surfaces}

Exterior surfaces are modelled as two thermal resistances with a centered heat capacitance between the exterior and zone $z$. Each surface $s$ is adjacent to exactly one zone $z$. The heat transfer across exterior surface $s$ is described by the balance equation for the exterior side:

$$
\dot{Q}_{s}^{c n v, e x t}+\dot{Q}_{s}^{i r r, e x t}-\dot{Q}_{s}^{e m s, s k y}-\dot{Q}_{s}^{e m s, g n d}=\dot{Q}_{s}^{e x t, s u r}
$$

Where $\dot{Q}_{s}^{c n v, e x t}$ is the convective heat transfer from the exterior towards surface $s, \dot{Q}_{s}^{i r r}$,ext is the incident irradiation onto surface $s, \dot{Q}_{s}^{e m s, s k y}$ and $\dot{Q}_{s}^{e m s, g n d}$ are the emitted radiation from surface $s$ towards the sky and the ground. The symbol $\dot{Q}_{s}^{e x t, s u r}$ describes the heat transfer from the exterior towards the center of the surface.

The balance equation for the interior side of surface $s$ is expressed as:

$$
\dot{Q}_{s}^{s u r, i n t}=\dot{Q}_{s, z}^{c n v, i n t}-\dot{Q}_{s}^{i r r, i n t}
$$

On the interior side, $\dot{Q}_{s}^{\text {sur,int }}$ is heat transfer from the center towards the interior side of the surface, $\dot{Q}_{s, z}^{c n v, i n t}$ is the convective heat transfer from the interior side of surface $s$ towards zone $z$ and $\dot{Q}_{s}^{\text {irr,int }}$ is the incident irradiation reaching surface $s$ through exterior windows adjacent to the same zone $z$.

The exterior convective term $\dot{Q}_{s}^{c n v, e x t}$ is expressed as:

$$
\dot{Q}_{s}^{c n v, e x t}=A_{s} h^{e x t}\left(T^{a m b}-T_{s}^{s u r, e x t}\right)
$$

Where $A_{s}$ is the surface area of surface $s$ and $h^{\text {ext }}$ is the exterior heat transfer coefficient which is given according to ISO 6946 as $h^{e x t}=\left(0.04 \mathrm{~m}^{2} \mathrm{~K} \mathrm{~W}^{-1}\right)^{-1}$. The symbol $T^{a m b}$ is the ambient temperature and $T_{s}^{\text {sur,ext }}$ is the exterior surface temperature of surface $s$.

The exterior irradiation term $\dot{Q}_{s}^{i r r, e x t}$ is expressed as:

$$
\dot{Q}_{s}^{i r r, e x t}=A_{s} \alpha_{s} \dot{q}_{d}^{i r r, e x t}, \quad d=d(s)
$$

Where $\alpha_{s}$ is the absorption coefficient of surface $s$ assuming a uniform absorption across the spectrum of the incident irradiation. The symbol $\dot{q}_{d}^{i r r, e x t}$ is the total incident irradiation onto a surface oriented towards direction $d=\{N, E, S, W\}$, i.e., vertically facing North $N$, East $E$, South $S$, West $W$ or horizontally facing upwards $H$, depending on the respective surface's orientation $d=d(s)$.

The exterior sky emission term $\dot{Q}_{s}^{e m s, s k y}$ describes the radiative heat loss through emission towards the sky. The term is expressed as:

$$
\dot{Q}_{s}^{e m s, s k y}=A_{s} h_{s}^{s k y}\left(T_{s}^{s u r, e x t}-T^{s k y}\right)
$$

In this linear approximation, the symbol $h_{s}^{s k y}$ is introduced as the sky heat transfer coefficient of surface $s$, whereas $T^{s k y}$ is the sky temperature. The sky heat transfer coefficient $h_{s}^{s k y}$ in turn is defined as:

$$
h_{s}^{s k y}=4 \sigma \varepsilon_{s} F_{d}^{s k y}\left(\frac{T_{s}^{s u r, e x t, l i n}+T^{s k y, l i n}}{2}\right)^{3}
$$

Where $\sigma, \varepsilon_{s}$ and $F_{d}^{s k y}$ are the Stefan-Boltzmann constant, the surface emission coefficient of surface $s$ for long-wave radiations and the view factor of direction $d(s)$ towards the sky. The temperatures $T_{s}^{\text {sur,ext,lin }}$ 
and $T^{\text {sky,lin }}$ are linearization constants that are defined as the average values of $T_{s}^{\text {sur,ext }}$ and $T^{\text {sky }}$. The term $F_{d}^{s k y}$ is defined as $F_{d}^{s k y}=0.5$ for $d \in$ $\{N, E, S, W\}$ and $F_{d}^{s k y}=1 d=H$.

The exterior ground emission term $\dot{Q}_{s}^{\text {ems,gnd }}$ radiative heat loss through emission towards the ground as well as the built environment. The term is expressed similar to $\dot{Q}_{s}^{e m s, s k y}$ as:

$$
\dot{Q}_{s}^{e m s, g n d}=A_{s} h_{s}^{g n d}\left(T_{s}^{s u r, e x t}-T^{a m b}\right)
$$

Where $h_{s}^{\text {gnd }}$ is introduced as the ground heat transfer coefficient of surface $s$, whereas $T^{a m b}$ is the ambient temperature. The ground heat transfer coefficient $h_{s}^{g n d}$ in turn is defined as:

$$
h_{s}^{g n d}=4 \sigma \varepsilon_{s} F_{d}^{g n d}\left(\frac{T_{s}^{s u r, e x t, l i n}+T^{a m b, l i n}}{2}\right)^{3}
$$

Where $F_{d}^{g n d}$ is the view factor of direction $d(s)$ towards the ground. The temperatures $T_{s}^{\text {sur,ext,lin }}$ and $T^{a m b, l i n}$ are linearization constants that are defined as the average values of $T_{s}^{s u r, e x t}$ and $T^{a m b}$. The term $F_{d}^{g n d}$ is defined as $F_{d}^{g n d}=0.5$ for $d \in\{N, E, S, W\}$ and $F_{d}^{g n d}=0 d=H$.

The interior convective term $\dot{Q}_{s, z}^{c n v, i n t}$ is expressed applying eq. 6 to $h_{d}^{\text {int }}, T_{s}^{\text {sur,int }}$ and $T^{z}$, which are the interior heat transfer coefficient, the interior surface temperature of surface $s$ and the zone air temperature. The interior heat transfer coefficient $h_{d}^{\text {int }}$ is defined according to ISO 6946 as $h_{d}^{\text {int }}=$ $\left(0.13 \mathrm{~m}^{2} \mathrm{~K} \mathrm{~W}^{-1}\right)^{-1}$ for $d \in\{N, E, S, W\}$ and $h_{d}^{\text {int }}=$ $\left(0.17 \mathrm{~m}^{2} \mathrm{~K} \mathrm{~W}^{-1}\right)^{-1}$ for $d=H$.

The interior irradiation term $\dot{Q}_{s}^{\text {irr,int }}$ is expressed applying eq. 7 to $\dot{q}_{z}^{\text {irr }, \text { int }}$, which is the interior irradiation incident to all surfaces of zone $z$. The interior radiation $\dot{q}_{z}^{i r r, i n t}$ is in fact the irritation which has entered zone $z$ by passing through adjacent windows and is assumed to be uniformly distributed to all surfaces. This term is expressed as:

$$
\dot{q}_{z}^{i r r, i n t}=\frac{\sum_{w \in \mathcal{W}_{z}} A_{w} \tau_{w} \dot{q}_{d(w)}^{i r r, e x t}}{\sum_{s \in \mathcal{S}_{z}} A_{s}}
$$

Where $\tau_{w}$ is the transmission coefficient of window $w$. The sets $\mathcal{W}_{z}$ and $\mathcal{S}_{z}$ contain all windows $w$ and surfaces $s$ that are adjacent to zone $z$.

Finally, the coupling terms $\dot{Q}_{s}^{\text {ext,sur }}$ and $\dot{Q}_{s}^{\text {sur,int }}$ are defined:

$$
\begin{aligned}
& \dot{Q}_{s}^{\text {ext,sur }}=A_{s} 2 h_{s}\left(T_{s}^{\text {sur }, \text { ext }}-T_{s}\right) \\
& \dot{Q}_{s}^{\text {sur }, \text { int }}=A_{s} 2 h_{s}\left(T_{s}-T_{s}^{\text {sur }, \text { int }}\right)
\end{aligned}
$$

Where $h_{s}$ is the heat transfer coefficient through surface $s$ and $T_{s}$ is the mean surface temperature, i.e., the temperature at the center of the surface $s$. For surfaces with a neglectable heat capacity, e.g., windows, the relationship simplifies to:

$$
\dot{Q}_{s}^{e x t, i n t}=A_{s} h_{s}\left(T_{s}^{s u r, e x t}-T_{s}^{s u r, i n t}\right)
$$

Equations (4) to (13) and (14) define an overdetermined equation system, such that the surface temperatures $T_{s}^{\text {sur,ext }}$ and $T_{s}^{\text {sur,int }}$ can be eliminated. The final equations are omitted here for the sake of brevity.

\section{Interior surfaces}

Interior surfaces are modelled by applying interior surface balance equation (eq. 5) for both sides of the surface.

\section{Infiltration}

The heat transfer towards zone $z$ due to infiltration $\dot{Q}_{z}^{i n f}$ is defined as:

$$
\dot{Q}_{z}^{i n f}=V_{z} C^{t h, a i r} n_{z}^{i n f}\left(T^{a m b}-T_{z}\right)
$$

Where $V_{z}$ is the volume of zone $z, C^{\text {th,air }}$ is the heat capacity of air and $n_{z}^{i n f}$ is the infiltration rate.

\section{Occupancy gains}

Assuming perfect knowledge of the building occupancy schedule, the heat transfer towards zone $z$ due to occupancy gains $\dot{Q}_{z}^{o c c}$, i.e., internal gains, is expressed as:

$$
\dot{Q}_{z}^{o c c}=A_{z} \dot{q}_{z}^{o c c}
$$

Where $A_{z}$ is the area of zone $z$ and $\dot{q}_{z}^{o c c}$ is the specific thermal gain due to occupancy.

\section{HVAC system}

The heat transfer towards zone $z$ from the HVAC system $\dot{Q}_{z}^{h v a c}$ is expressed as:

$$
\dot{Q}_{z}^{h v a c}=\eta^{h v a c} P_{z}^{h v a c, e l}
$$

Where $\eta^{\text {hvac }}$ is the efficiency factor of the HVAC system and $P_{z}^{h v a c, e l}$ is the electric power consumption of the HVAC system associated with thermal demand supplied at zone $z$.

\section{State space model}

The state space formulation of the thermal building model is obtained by arranging the model variables into vectors and the model parameters into the appropriate matrices:

$$
\begin{aligned}
\dot{\boldsymbol{x}} & =\boldsymbol{A} \boldsymbol{x}+\boldsymbol{B}_{u} \boldsymbol{u}+\boldsymbol{B}_{v} \boldsymbol{v} \\
\boldsymbol{y} & =\boldsymbol{C} \boldsymbol{x}+\boldsymbol{D}_{u} \boldsymbol{u}+\boldsymbol{D}_{v} \boldsymbol{v}
\end{aligned}
$$

The matrices $\boldsymbol{A}, \boldsymbol{C}$ are the state and output matrix, and $\boldsymbol{B}_{u}, \boldsymbol{D}_{u}, \boldsymbol{B}_{v}, \boldsymbol{D}_{v}$ are the input and feed-through matrix, on the control and disturbance vector respectively. The vectors $\boldsymbol{x}, \boldsymbol{u}, \boldsymbol{v}, \boldsymbol{y}$ are the state, input, 
disturbance and output vectors. The model variables are arranged into the vectors as follows:

$$
\begin{aligned}
\boldsymbol{x} & =\left[T_{z}, T_{s}\right]^{\top} \\
\boldsymbol{u} & =\left[P_{z}^{H V A C, e l}\right]^{\top} \\
\boldsymbol{v} & =\left[T^{a m b}, \dot{q}_{d}^{i r r}, \dot{q}_{z}^{o c c}\right]^{\top} \\
\boldsymbol{y} & =\left[T_{z}, p_{z}^{H V A C, e l}\right]^{\top}
\end{aligned}
$$

Lastly, the discrete thermal building model in the state space form is obtained by application of zeroorder hold discretization:

$$
\begin{aligned}
\boldsymbol{x}_{t+1} & =\boldsymbol{A}_{t} \boldsymbol{x}_{t}+\boldsymbol{B}_{u, t} \boldsymbol{u}_{t}+\boldsymbol{B}_{v, t} \boldsymbol{v}_{t} \\
\boldsymbol{y}_{t} & =\boldsymbol{C}_{t} \boldsymbol{x}_{t}+\boldsymbol{D}_{u, t} \boldsymbol{u}_{t}+\boldsymbol{D}_{v, t} \boldsymbol{v}_{t}
\end{aligned}
$$

Where ()$_{t}$ denotes variable instances at time step $t$. Note that all matrices are time-dependent to permit the use of time-dependent linearization parameters.

\section{Demand controlled ventilation}

In the first part of this section, the baseline fixed ventilation strategy is introduced. Then, two DCV strategies are presented: an occupancy-based strategy developed from international standards and an IAQ-based strategy developed along with an IAQ model.

\section{Fixed ventilation strategy}

Ventilation in commercial buildings is meant to ensure good indoor air quality (IAQ), which has significant effects on productivity at work and occupants' health (Satish et al. (2015); Fanger (2006)). In conservative ventilation strategies, the outdoor airflow rate is fixed based on the designed occupancy rate of the respective zone and does not depend on the actual occupancy rate during operation. Therefore, HVAC systems have to constantly provide a fixed amount of outdoor air. This strategy is defined by constraining the outdoor airflow rate $\dot{V}_{z}$ in zone $z$ by a fixed minimum requirement $\dot{V}_{z}^{\text {min }}$, which is the minimum outdoor airflow rate in the zone to maintain the desired IAQ:

$$
\dot{V}_{z} \geq \dot{V}_{z}^{\min }
$$

\section{Occupancy-based strategy}

The purpose of outdoor air ventilation is to dilute indoor pollutants, which can be emitted either from (1) occupants and (2) building materials. ASHRAE Standard 62.1 defines the minimum outdoor airflow rate according to the building occupancy and the building size, as a sum to account for both kinds of pollution. The occupancy-based strategy is defined by constraining the ventilation rate at zone according to:

$$
\dot{V}_{z, t} \geq \dot{V}_{z, t}^{\min , o c c}=\dot{v}^{o c c} n_{z}^{o c c}+\dot{v}^{b l g} A_{z}
$$

Where $\dot{v}^{o c c}, \dot{v}^{b l g}$ are the specfic outdoor airflow rate per person and per area respectively. The symbols $n_{z, t}^{o c c}$ and $A_{z}$ are number of occupants in the zone $z$ at time step $t$ and the floor area of zone $z$. Values for $\dot{v}^{o c c}$ and $\dot{v}^{b l g}$ are derived according to ASHRAE Standard 62.1. With this strategy, the minimum outdoor airflow rate $\dot{V}_{z, t}^{\text {min }, o c c}$ varies dynamically with the occupancy.

\section{IAQ-based strategy}

IAQ modelling is particularly challenging due to the number of indoor pollutants found in buildings (Fanger (2006)). Studies have quantified the impact on human health only for a few single indoor pollutants, and those impacts may be enhanced when the pollutants are combined. However, a simplified model can be formulated by understanding the correlations within pollutants with similar behaviour, which can then be clustered according to Zhu and Li (2017) into: (1) pollutants from outdoors (respiratory particles), (2) pollutants from building materials (Formaldehyde, HCHO) and (3) pollutants from human metabolism $\left(\mathrm{CO}_{2}\right.$, microorganisms). Pollutants from group (1) are treated with particle filters and other techniques and do not influence the outdoor airflow requirement, whereas pollutants from group (2) and (3) are diluted through outdoor air ventilation. Since pollutants from group (2) are related to building materials, this paper assumes a constant requirement to remove such pollutants which only depends on the zone area $A_{z}$, similar to eq. 22 :

$$
\dot{V}_{z}^{\text {min }, b l d}=\dot{v}^{b l d} A_{z}
$$

Since pollutants within each cluster are correlated, one pollutant can be chosen as the reference for each cluster (Zhu and Li (2017)). The reference pollutant's behaviour would depict the group's behaviour and consequently the current level of IAQ. In our model, $\mathrm{CO}_{2}$ is chosen as the reference pollutant for group (3). ASHRAE Standard 62.1 defines the maximum $\mathrm{CO}_{2}$ concentration to ensure good IAQ at $700 \mathrm{ppm}$ above outdoor concentration, i.e. around $1100 \mathrm{ppm}$ when considering an outdoor $\mathrm{CO}_{2}$ concentration of 400 ppm.

In office buildings, the main source for $\mathrm{CO}_{2}$ is human activity. The $\mathrm{CO}_{2}$ generation rate per person $G$ depends on the basal metabolic rate, which varies with age, sex and body mass, of the occupants and their level of physical activity. For office work, $G$ ranges between $0.0041 \mathrm{~L} / \mathrm{s}$ and $0.0056 \mathrm{~L} / \mathrm{s}$ (Persily and de Jonge (2017)). Assuming a large number of occupants, this paper approximates the mean $\mathrm{CO}_{2}$ generation rate for office work at $0.0049 \mathrm{~L} / \mathrm{s}$. Assuming constant air density, the mass balance for $\mathrm{CO}_{2}$ at zone $z$ volume yields:

$$
V_{z} \frac{\mathrm{d} c_{z}^{\mathrm{CO}_{2}}}{\mathrm{~d} t}=G n_{z}^{o c c}+\dot{V}_{z} c^{\mathrm{CO}_{2}, a m b}-\dot{V}_{z} c_{z}^{\mathrm{CO}_{2}}
$$




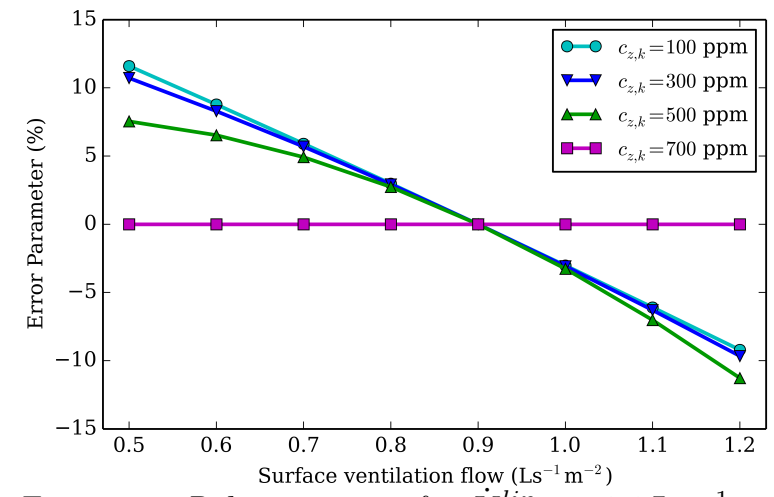

Figure 1: Relative error for $\dot{V}_{z}^{\text {lin }}=0.9 \mathrm{~L} \mathrm{~s}^{-1} \mathrm{~m}^{-2}$, $\Delta t=30 \mathrm{~min}$.

Where $V_{z}$ is the zone volume and $c^{\mathrm{CO}_{2}, a m b}, c_{z}^{\mathrm{CO}_{2}}$ are the concentration of $\mathrm{CO}_{2}$ in the ambient outdoor air and the zone air respectively. For the sake of brevity, this paper defines $c_{z}^{\mathrm{CO}_{2}}$ as the $\mathrm{CO}_{2}$ concentration in zone air minus the $\mathrm{CO}_{2}$ concentration in the ambient outdoor air. The number of occupants $n_{z}^{o c c}$ can be determined either with a attendance counter, with an indoor positioning system or even with $\mathrm{CO}_{2}$ sensors that derive the occupancy from measured concentrations (Ng et al. (2011); Wang et al. (2017)).

The $\mathrm{CO}_{2}$ model (eq. 24) is linearized according to:

$$
\begin{aligned}
\frac{\mathrm{d} c_{z}^{\mathrm{CO}_{2}}}{\mathrm{~d} t}=\frac{G n_{z}^{o c c}}{V_{z}} & -\frac{\dot{V}_{z}^{l i n} c_{z}^{\mathrm{CO}_{2}}}{V_{z}} \\
& -\frac{\dot{V}_{z} c_{z}^{\mathrm{CO}_{2}, l i n}}{V_{z}}+\frac{\dot{V}_{z}^{l i n} c_{z}^{\mathrm{CO}_{2}, l i n}}{V_{z}}
\end{aligned}
$$

Where $\dot{V}_{z}^{\text {lin }}$ and $c_{z}^{\mathrm{CO}_{2}, \text { lin }}$ are the linearization points for the outdoor airflow rate and the $\mathrm{CO}_{2}$ concentration.

The linearized IAQ model can then be incorporated into the building model's state-space equation (20) and discretized along with it. The optimization problem (eq. 2) is completed with the IAQ constraints $c_{z}^{\mathrm{CO}_{2}} \leq c^{\mathrm{CO}_{2}, \max }$ and $\dot{V}_{z} \geq \dot{V}_{z}^{\text {min,bld }}$.

\section{IAQ modelling error}

According to ASHRAE Standard 62.1, good IAQ is ensured for $\mathrm{CO}_{2}$ concentrations varying from 0 to 700 ppm above ambient $\mathrm{CO}_{2}$ concentration. Because of the large interval of allowable $\mathrm{CO}_{2}$ concentrations, the linearized model (eq. 26) may yield nonneglectable errors.

As a starting point, the linearized, discretized $\mathrm{CO}_{2}$ model is expressed as:

$$
\begin{aligned}
\hat{c}_{z, t+1}^{\mathrm{CO}_{2}} & =\hat{c}_{z, t}^{\mathrm{CO}_{2}}+\left(\mathrm{e}^{-\frac{\dot{V}_{z}^{l i n}}{V_{z}} \cdot \Delta t}-1\right) \\
& \cdot\left(\hat{c}_{z, t}^{\mathrm{CO}_{2}}+\frac{c^{\mathrm{CO}_{2}, l i n}}{\dot{V}_{z}^{l i n}} \cdot \dot{V}_{z, t}-c^{\mathrm{CO}_{2}, l i n}-\frac{G n_{z}^{o c c, l i n}}{\dot{V}_{z}^{l i n}}\right)
\end{aligned}
$$

Let $\epsilon$ be the relative error around the linearization point $\dot{V}_{z}^{\text {lin }}$. A first order approximation around $\dot{V}_{z}^{\text {lin }}$ yields:

$$
\begin{aligned}
& \epsilon=\frac{c_{z, t+1}^{\mathrm{CO}_{2}}-\hat{c}_{z, t+1}^{\mathrm{CO}_{2}}}{\dot{V}_{z, t}-\dot{V}_{z}^{l i n}}=\frac{\mathrm{e}^{-\frac{\dot{V}_{z}^{l i n}}{V_{z}} \cdot \Delta t}}{\dot{V}_{z}^{l i n}}\left(\frac{G n_{z, t}^{o c c}}{\dot{V}_{z}^{l i n}}-c^{\mathrm{CO}_{2}, l i n}\right) \\
& +\mathrm{e}^{-\frac{\dot{V}_{z}^{l i n}}{V_{z}} \cdot \Delta t} \frac{\Delta t}{V_{z}}\left(\frac{G n_{z, t}^{o c c}}{\dot{V}_{z}^{l i n}}-c_{z, t}^{\mathrm{CO}_{2}}\right) \\
& -\frac{1}{\dot{V}_{z}^{l i n}}\left(\frac{G n_{z, t}^{o c c}}{\dot{V}_{z}^{l i n}}-c^{\mathrm{CO}_{2}, l i n}\right)
\end{aligned}
$$

From eq. 27, the linearization point $c^{\mathrm{CO}_{2}, \text { lin }}$ is defined such that the latter terms of eq. 27 vanish:

$$
c^{\mathrm{CO}_{2}, l i n}=\frac{G n_{z}^{o c c, l i n}}{\dot{V}_{z}^{l i n}}
$$

Where $n_{z}^{o c c, l i n}$ becomes the linearization point for the number of occupants, such that the model remains linear. The resulting error is depicted in fig. 1 . The chosen linearization approach for the IAQ model is only suited for small variations in the CO2 concentration. To mitigate the dependency on the chosen linearization point, a robust optimization approach can be considered, but is excluded from this paper for the sake of clarity and brevity.

\section{Flexibility indicators}

This section introduces three flexibility indicators in order to quantify the change in DSF due to demand controlled ventilation strategies. Three kinds of flexibility indicators are presented: forced operation flexibility, deviation flexibility and reserves.

\section{Forced operation flexibility}

Since DSF is intended to shift loads from one time period to another, the first indicator quantifies the maximum load that can be deferred for a given time period while evolving within the system operation constraints.

Let $\boldsymbol{u}^{\text {bas }}$ be the baseline load schedule, which is the energy optimal operation, solution of eq. 2 . The time period considered for estimating flexibility is $\left[t_{1}, t_{2}\right]$, and the flexibility indicator $f_{t_{1}, t_{2}}$ is the maximum percentage of the baseline that can be shifted over the time period $\left[t_{1}, t_{2}\right]$, i.e., it is shifted from $t_{1}$ towards $t_{2}$. The optimization problem to determine $f_{t_{1}, t_{2}}$ is formulated as:

$$
\begin{array}{cl}
\max _{\boldsymbol{u}_{t}} & f_{t_{1}, t_{2}} \\
\text { s.t. } & (2 \mathrm{a}),(20) \\
& \left\|\boldsymbol{u}_{t}\right\|=\left(1-f_{t_{1}, t_{2}}\right)\left\|\boldsymbol{u}_{t}^{\text {bas }}\right\| \quad \forall t \in\left[t_{1}, t_{2}\right]
\end{array}
$$

This procedure can be iterated and averaged over different time periods to obtain an average value of the 
flexibility indicator.

\section{Deviation flexibility}

Considering indirect control through energy prices, a flexible load operator will determine its operation schedule by minimizing its energy costs (as in eq. 2). Therefore, the deviation flexibility describes the price sensitivity of the flexible load, i.e. how the load reacts to price changes. Assuming the nominal operations are obtained with a flat price, the flexibility indicator is defined as the load variation at time step $t$ in response to a change in energy price at $t$.

Let $\boldsymbol{u}_{t}^{\text {nom }}$ be the nominal operations obtained with a flat price, and $\boldsymbol{u}_{\Delta c, t}$ the optimal operations after a change in energy prices $\Delta c$ at time step $t$. The flexibility indicator $d_{\Delta c, t}$ is defined as:

$$
d_{\Delta c, t}=\frac{\left\|\boldsymbol{u}_{\Delta c, t}\right\|-\left\|\boldsymbol{u}_{t}^{n o m}\right\|}{\left\|\boldsymbol{u}_{t}^{n o m}\right\|}
$$

This procedure can be iterated over $t$ and $\Delta c$ and averaged over $t$.

\section{Reserve flexibility}

Due to their thermal inertia, buildings are able to vary their power consumption rapidly with minor changes on the comfort, and therefore to provide high-quality demand-side reserves. For a given reserve price, buildings would schedule their load and reserves, and the load would be curtailed if the reserves are called upon. Under the Demand Response programme of NEMS, the building would be paid for the availability of reserves, rather than on their activation.

An optimization problem can be formulated to schedule reserves along with the loads. Let $\boldsymbol{u}^{c}$ and $\boldsymbol{u}^{n c}$ be the curtailed and non-curtailed loads respectively. In Singapore, the scheduled reserves are reserves down, therefore the amount of reserves paid is the difference between the non-curtailed and the curtailed load. The new cost of operations $j_{u, r}$ can be modeled accordingly.

$$
\begin{aligned}
j_{u, r}(\boldsymbol{u}) & =\sum_{t \in \mathcal{T}}\left(c_{t}^{e l c}\left\|\boldsymbol{u}_{t}^{n c}\right\|-c_{t}^{r e s} r_{t}\right) \Delta t \\
r_{t} & =\left\|\boldsymbol{u}_{t}^{n c}\right\|-\left\|\boldsymbol{u}_{t}^{c}\right\|
\end{aligned}
$$

Scheduled reserves $r_{t}$ are paid at the reserve price $c^{r e s}$. The optimal reserve scheduling problem ensures that both the non-curtailed $n c$ and the curtailed $c$ trajectories remain within the constraints:

$$
\begin{array}{ll}
\min _{\boldsymbol{u}_{t}, r_{t}} & j_{u, r}(\boldsymbol{u}) \\
\mathrm{s.t.} & \boldsymbol{x}_{t+1}^{c}=\boldsymbol{A}_{t} \boldsymbol{x}_{t}^{n c}+\boldsymbol{B}_{u, t} \boldsymbol{u}_{t}^{c}+\boldsymbol{B}_{v, t} \boldsymbol{v}_{t} \\
& \boldsymbol{y}_{t}^{c}=\boldsymbol{C}_{t} \boldsymbol{x}_{t}^{c}+\boldsymbol{D}_{u, t} \boldsymbol{u}_{t}^{c}+\boldsymbol{D}_{v, t} \boldsymbol{v}_{t} \\
& \boldsymbol{x}_{t+1}^{n c}=\boldsymbol{A}_{t} \boldsymbol{x}_{t}^{n c}+\boldsymbol{B}_{u, t} \boldsymbol{u}_{t}^{n c}+\boldsymbol{B}_{v, t} \boldsymbol{v}_{t} \\
& \boldsymbol{y}_{t}^{n c}=\boldsymbol{C}_{t} \boldsymbol{x}_{t}^{n c}+\boldsymbol{D}_{u, t} \boldsymbol{u}_{t}^{n c}+\boldsymbol{D}_{v, t} \boldsymbol{v}_{t} \\
& \boldsymbol{y}_{\min } \leq \boldsymbol{y}_{t}^{c} \leq \boldsymbol{y}_{\max } \\
& \boldsymbol{y}_{\min } \leq \boldsymbol{y}_{t}^{n c} \leq \boldsymbol{y}_{\max } \\
& r_{t}=\left\|\boldsymbol{u}_{t}^{n c}\right\|-\left\|\boldsymbol{u}_{t}^{c}\right\|
\end{array}
$$

The vectors $\boldsymbol{x}_{t}^{c}, \boldsymbol{y}_{t}^{c}, \boldsymbol{x}_{t}^{n c}$ and $\boldsymbol{y}_{t}^{n c}$ are the curtailed and non-curtailed state and output vectors at time step $t$, respectively. The flexibility indicator $r^{*}$ is defined as:

$$
r_{c^{e l c}, c^{r e s}, t}^{*}=\frac{r_{t}}{\left\|\boldsymbol{u}_{t}^{b a s}\right\|}
$$

The procedure can be iterated over $t$ and $c^{r e s}$ and averaged over $t$.

\section{Test Case}

The presented DCV startegies are evaluated for one storey of approx. $750 \mathrm{~m}^{2}$ at the CREATE Tower in Singapore. The level is entirely occupied by an openplan office.

\section{Thermal model parameters}

The geometric information of zones and surfaces are derived from the architectural drawings of the test case. Based on the building documentation, the thermal building model parameters in table 1 are defined. The number of occupants $\dot{n}_{z}^{\text {occ }}$ and occupancy gains $\dot{q}_{z}^{\text {occ }}$ have been recorded for the test period.

Table 1: Thermal model parameters.

\begin{tabular}{|c|c|}
\hline Parameter & Value \\
\hline$h_{s}$ (ext./int. surf. type 1) & $0.54 \mathrm{~W} \mathrm{~K}^{-1} \mathrm{~m}^{-2}$ \\
\hline$h_{s}$ (ext./int. surf. type 2$)$ & $3.85 \mathrm{~W} \mathrm{~K}^{-1} \mathrm{~m}^{-2}$ \\
\hline$\alpha_{s}$ (all surfaces) & 0.3 \\
\hline$\varepsilon_{s}$ (all surfaces) & 0.87 \\
\hline$\tau_{w}$ (ext. windows) & 0.2 \\
\hline$C_{z}^{\text {th }}$ & $1.21 \mathrm{~kJ} \mathrm{~K}^{-1} \mathrm{~m}^{-3}$ \\
\hline$C_{s}^{t h}$ (ext./int. surf. type 1$)$ & $91.5 \mathrm{~kJ} \mathrm{~K}^{-1} \mathrm{~m}^{-3}$ \\
\hline$C_{s}^{t h}$ (ext./int. surf. type 2$)$ & $810 \mathrm{~kJ} \mathrm{~K}^{-1} \mathrm{~m}^{-3}$ \\
\hline$n_{z}^{\text {inf }}$ & $0.2 \mathrm{~h}^{-1}$ \\
\hline$\eta^{\text {hvac }}$ & 4 \\
\hline$T_{s}^{\text {sur,lin }}$ & $35^{\circ} \mathrm{C}$ \\
\hline$T^{\text {sky,lin }}$ & $17^{\circ} \mathrm{C}$ \\
\hline$T^{\text {amb,lin }}$ & $30^{\circ} \mathrm{C}$ \\
\hline
\end{tabular}

\section{IAQ model parameters}

Parameter values for the DCV startegies and IAQ model are chosen according to table 2 . 
Table 2: IAQ model parameters.

\begin{tabular}{|c|c|}
\hline Parameter & Value \\
\hline$\dot{v}^{\text {occ }}$ & $2.5 \mathrm{~L} / \mathrm{s} /$ person \\
\hline$\dot{v}^{b l g}$ & $0.3 \mathrm{~L} \mathrm{~s}^{-1} \mathrm{~m}^{-2}$ \\
\hline$n_{z}^{\text {occlin }}$ & 90 \\
\hline$\dot{V}_{z}^{\text {lin }}$ & $0.9 \mathrm{~L} \mathrm{~s}^{-1} \mathrm{~m}^{-2}$ \\
\hline
\end{tabular}

\section{Weather data}

Weather data is obtained from a nearby weather station at the campus of the National University of Singapore (NUS). The sky temperature $T^{s k y}$ is defined by an approximation for tropical climate as $T^{s k y}=T^{a m b}-13 \mathrm{~K}$ according to ISO 52016-1.

\section{Energy price data}

The electricity price and reserve price of the National Electricity Market of Singapore (NEMS) from 2 January 2017 are used for this test case (Figure 2). For the deviation flexibility indicator the mean value of the electricity price is taken as the flat price.

\section{Comfort constraints}

The thermal comfort and IAQ constraints are given in table 3. For DCV strategies, also refer to the respectively defined constraints above.

Table 3: Comfort constraints.

\begin{tabular}{|c|c|c|}
\hline Parameter & Min. value & Max. value \\
\hline$c_{z}^{\mathrm{CO}_{2}}$ & - & $700 \mathrm{ppm}$ \\
\hline$T_{z}$ & $20^{\circ} \mathrm{C}$ & $25^{\circ} \mathrm{C}$ \\
\hline
\end{tabular}

\section{Results and discussion}

The building energy consumption over a day for each of the three different strategies are given in Table 4 . Energy savings from DCV approaches are approximately of $55 \%$ versus the fixed ventilation strategy, which is coherent with the $5 \%$ to $80 \%$ range of savings that can be found in literature (Schell and Inthout (2001)).

Table 4: Total electricity consumption over $24 \mathrm{~h}$.

\begin{tabular}{|c|c|c|}
\hline No DCV & $\begin{array}{c}\text { Occ.-based } \\
\text { DCV }\end{array}$ & $\begin{array}{c}\text { IAQ-based } \\
\text { DCV }\end{array}$ \\
\hline $353 \mathrm{Wh} / \mathrm{m}^{2}$ & $149 \mathrm{Wh} / \mathrm{m}^{2}$ & $169 \mathrm{Wh} / \mathrm{m}^{2}$ \\
\hline
\end{tabular}

Figure 4 exhibits a range of price variations for which the deviation flexibility indicator remains at zero, which indicates that there is no change in the building load schedule. Because of the losses, a shifted load increases in size, and consequently the cost rises. Therefore, it may not be economically interesting to shift a load for a small $\Delta c$, as the increase in load due to losses would be more expensive than the expected gains. The price variation that triggers the load shifting is hence of high interest to quantify how flexible a building is. As the scheduling of reserves involves load shifting as well, Figure 4 exhibits a reserve price below which reserves are not scheduled.

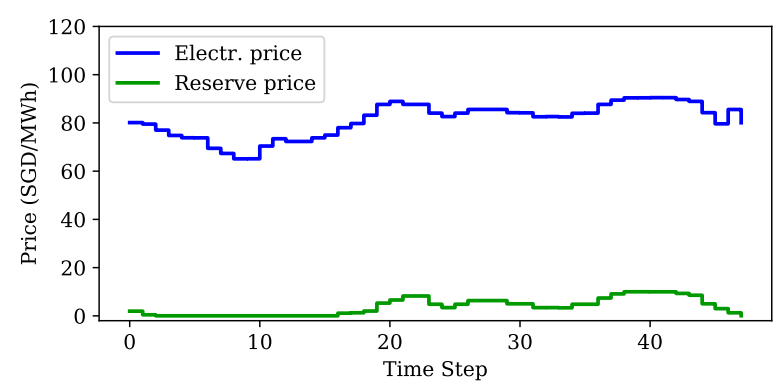

Figure 2: Prices from NEMS.

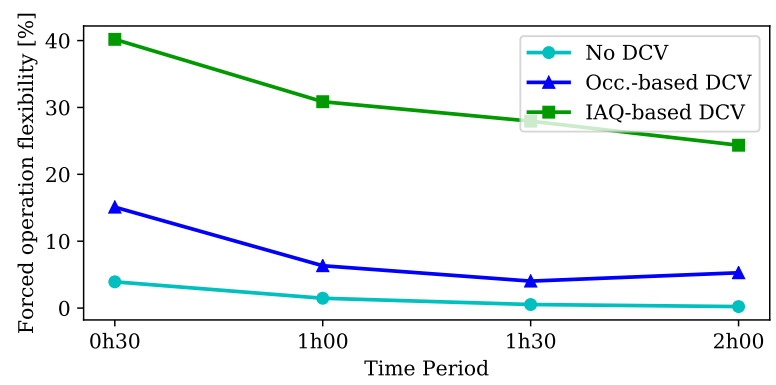

Figure 3: Forced flexibility indicator.

Figures 3, 4 and 5 depict the estimated flexibility indicators for the three control strategies. The IAQbased DCV strategies proves to be the most flexible for each indicator. This strategy is the one that needs the smallest price variation to trigger a change in the loads (Figure 4), as well as being the one that schedules reserves at the smallest reserve price (Figure 5).

The occupancy-based DCV strategy and the fixed ventilation strategy are both defining a minimum level of ventilation, instead of an IAQ allowable range. Therefore ventilation loads are fixed at this minimum value, and are not able to be shifted over time. For instance, these two strategies provide similar amounts of reserves: the different values of their reserve indicator is mainly a consequence of the $58 \%$ difference in their baseline.

The analysis of reserves scheduling with high reserve prices reveals two side effects. First, reserves scheduling induces a non-neglectable change in the baseline load schedule. The IAQ-based DCV strategy combined with a reserve price multiplier of 5 provides $27 \%$ of the baseline as reserves, along with an $11 \%$ increase in the baseline. Indeed, DSF is provided at the expense of energy consumption. Second, the reserve price shall remain lower than the energy price.

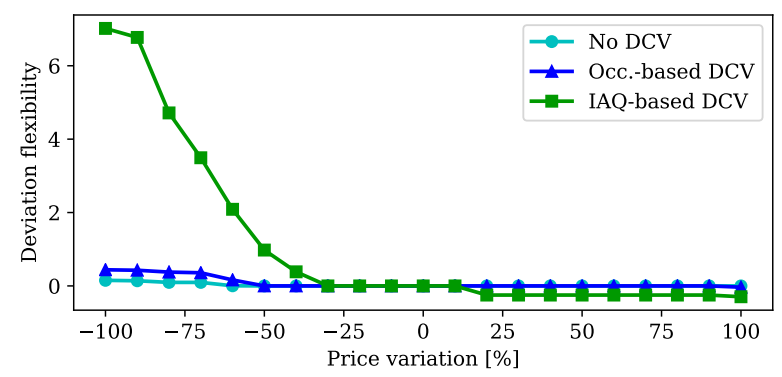

Figure 4: Deviation flexibility indicator. 


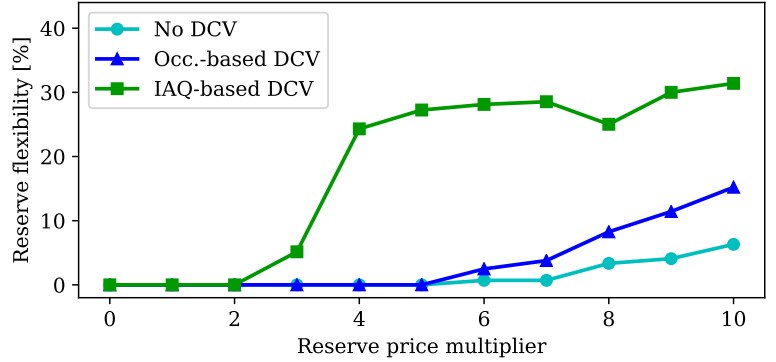

Figure 5: Reserve flexibility indicator.

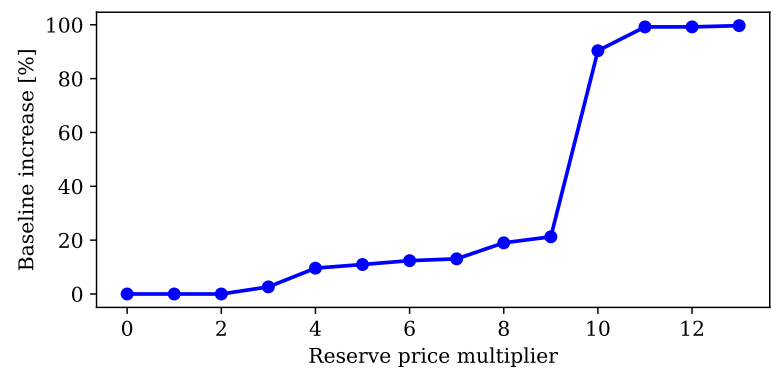

Figure 6: Baseline variation with reserve price.

In the opposite case, the building optimal set of operations is to use as much energy as possible, which would be counterproductive in providing flexibility to the grid. The sudden baseline increase obtained for a reserve price higher than the energy price is shown Figure 6. Once the energy price is reached, the building maximizes its energy consumption, which is in this example twice as high as the regular optimal energy consumption.

\section{Conclusion}

This paper introduced methods for increasing the DSF of commercial buildings with DCV, by considering ventilation as a comfort constraint rather than as a fixed requirement. Two DCV strategies were defined and the change in DSF for each strategy was evaluated. As indicators for DSF, the forced operation, price sensitivity and reserve scheduling indicators were formulated. The IAQ-based DCV strategy has proved to be the strategy which provides the most DSF. Therefore, buildings operators which are looking into DSF-enabled building control should consider the installation of IAQ sensing or occupancy detection equipment such that a higher DSF can be utilized.

\section{Acknowledgment}

This work was financially supported by the Singapore National Research Foundation under its Campus for Research Excellence And Technological Enterprise (CREATE) programme.

\section{Nomenclature}

Let $\mathbb{R}$ be the domain of real numbers. Non-bold letters $x, X$ denote scalars $\mathbb{R}^{1 \times 1}$, bold lowercase letters $\boldsymbol{x}$ denote vectors $\mathbb{R}^{n \times 1}$ and bold uppercase letters $\boldsymbol{X}$ denote matrices $\mathbb{R}^{n \times m}$. The numbers $\mathbf{0}$ and $\mathbf{1}$ denote vectors or matrices of zeros and ones of appropriate size. The transpose of a vector or matrix is denoted by ()$^{\top}$. Symbols for physical properties are aligned with ISO 80000.

\section{References}

Atia, R. and N. Yamada (2016). Sizing and Analysis of Renewable Energy and Battery Systems in Residential Microgrids. IEEE Transactions on Smart Grid 7(3), 1204-1213.

Chua, K. J., S. K. Chou, W. M. Yang, and J. Yan (2013). Achieving better energy-efficient air conditioning - A review of technologies and strategies. Applied Energy 104, 87-104.

Fanger, P. O. (2006). What is IAQ? Indoor Air 16(5), 328-334.

Hanif, S., T. Massier, H. Gooi, T. Hamacher, and T. Reindl (2017). Cost Optimal Integration of Flexible Buildings in Congested Distribution Grids. IEEE Transactions on Power Systems 32(3), 2254-2266.

Laverge, J., N. Van Den Bossche, N. Heijmans, and A. Janssens (2011). Energy saving potential and repercussions on indoor air quality of demand controlled residential ventilation strategies. Building and Environment 46(7), 1497-1503.

Ng, M. O., M. Qu, P. Zheng, Z. Li, and Y. Hang (2011). CO2-based demand controlled ventilation under new ASHRAE Standard 62.1-2010: A case study for a gymnasium of an elementary school at West Lafayette, Indiana. Energy and Buildings 43(11), 3216-3225.

Pantazaras, A., M. Santamouris, S. E. Lee, and M. N. Assimakopoulos (2018). A decision tool to balance indoor air quality and energy consumption: A case study. Energy and Buildings 165, 246-258.

Persily, A. and L. de Jonge (2017). Carbon dioxide generation rates for building occupants. Indoor Air 27(5), 868-879.

Satish, U., W. B. Fisk, M. J. Mendell, K. Eliseeva, T. Hotchi, D. Sullivan, L. B. Cleckner, K. Shekhar, and K. Teng (2015). Impact of CO2 on human decision making and productivity. Journal of Applied Social Psychology 23, 847-866.

Schell, M. and D. Inthout (2001). Demand Control Ventilation Using CO2. ASHRAE Journal 43(2), 18-29.

Wang, W., Y. Lu, G. Huang, X. Luo, and J. Chen (2017). HVAC Energy Saving in IPS-enabled Large Space: An Occupancy Distribution Based Demand-driven Control Approach. Energy Procedia 105, 2083-2088.

Zhu, C. and N. Li (2017). Study on Grey Clustering Model of Indoor Air Quality Indicators. Procedia Engineering 205, 2815-2822. 